\title{
Accurate Prediction of Nonadiabatic Transitions through Avoided Crossings
}

\author{
Volker Betz and Benjamin D. Goddard \\ Mathematics Institute, University of Warwick, Coventry CV47AL, United Kingdom
}

(Received 7 August 2009; published 17 November 2009)

\begin{abstract}
In the case of one nuclear degree of freedom, we derive an explicit formula for the nuclear wave function transmitted through an avoided crossing, and show that it agrees to high accuracy with precise numerical calculations.
\end{abstract}

The time-dependent Born-Oppenheimer approximation (BOA) is at the basis of our understanding of dynamics of molecules. The small ratio $\varepsilon^{2}$ of electronic and nuclear mass allows one to replace the electronic degrees of freedom with an effective potential, and to separate the nuclear dynamics according to the electronic energy surfaces. This dramatically reduces the complexity of the problem.

Despite its success, situations where the BOA fails are of great interest in quantum chemistry. These occur when electronic energy levels are not well separated for a given nuclear configuration. Important applications include photodissociation of diatomic molecules like $\mathrm{NaI}$ [1], or the reception of light in the retina [2]. Two basic types of failure occur: conical crossings as appearing in [2], and avoided crossings. The latter are typical for systems with one nuclear degree of freedom [1,3], and are the topic of this Letter. We give an explicit formula, cf., (11), for the transmitted wave function at a generic avoided crossing, using only data that is local in time and space. An algorithm for calculating this wave function is then straightforward.

The importance of nonadiabatic transitions has led to many efforts to understand and predict them. A simplification of the problem is to replace the nuclear degree of freedom by a classical trajectory. It is both ancient [4] and well understood [5,6], and leads to the famous LandauZener (LZ) formula for the transition probabilities between electronic levels. This formula lies at the basis of several surface hopping models, such as [7-9]. While these and other [10] trajectory based methods yield reasonably good transition probabilities, their accuracy in predicting the shape of the transmitted wave function is limited [7]. An improvement to the LZ-transition rates, based on the full quantum scattering theory of the problem, is achieved by the Zhu-Nakamura theory [11]. Again, only transition rates are treated, and not the full transmitted wave function. Hagedorn and Joye [12] derive rigorous asymptotic formulas for the transmitted wave function, in the limit of $\varepsilon \rightarrow 0$. However, these formulas are difficult to apply in practice and are neither local in time nor space.

We consider a two level system with a Hamiltonian with one effective degree of freedom: $H=-(1 / 2) \varepsilon^{2} \partial_{x}^{2} \boldsymbol{I}+$ $V(x)$, where $V(x)=X(x) \sigma_{x}+Z(x) \sigma_{z}+d(x) \boldsymbol{I}$ is the real-symmetric potential energy matrix in the diabatic representation. $\boldsymbol{I}$ is the $2 \times 2$ unit matrix, and $\sigma_{x}, \sigma_{z}$ are the Pauli matrices. Units are such that $\hbar=1$ and the electron mass $m_{\mathrm{el}}=1 . \varepsilon^{2}$ is the ratio of electron and reduced nuclear mass.

The time-dependent Schrödinger equation is given by $i \varepsilon \partial_{t} \psi(x, t)=H \psi(x, t)$, with $\psi \in L^{2}\left(\mathbb{R}, \mathbb{C}^{2}\right)$, in the time scale where the nuclei move a distance of order one within a time of order one. It is convenient to switch to the adiabatic representation: let $U_{0}(x)$ diagonalize $V(x)$ for each $x$, and define $\psi_{0}=U_{0} \psi$. Then $\psi_{0}$ solves

$$
i \varepsilon \partial_{t} \psi_{0}=H_{0} \psi_{0}
$$

with $H_{0}$ given to leading order by

$$
H_{0}=-\frac{\varepsilon^{2}}{2} \partial_{x}^{2} \boldsymbol{I}+\left(\begin{array}{ll}
\rho(x)+d(x) & -\varepsilon \kappa_{1}(x)\left(\varepsilon \partial_{x}\right) \\
\varepsilon \kappa_{1}(x)\left(\varepsilon \partial_{x}\right) & -\rho(x)+d(x)
\end{array}\right) .
$$

Above, $\rho=\sqrt{X^{2}+Z^{2}}$ is half the energy level separation, and $\kappa_{1}=\left(X^{\prime} Z-Z^{\prime} X\right) /\left(Z^{2}+X^{2}\right)$ is the adiabatic coupling element. A consequence of the choice of time scale in (1) is that solutions will oscillate with frequency $1 / \varepsilon$. Thus the operator $\varepsilon \partial_{x}$ is actually of order one.

Generically, the entries of $V$ are analytic in the nuclear coordinate $x$, and eigenvalues of $V$ do not cross [3]. An avoided crossing is a (local or global) minimum of $\rho(x)$. Our aim is to describe the nonadiabatic transitions: Assume that (1) is solved with an initial wave packet that is fully in the upper adiabatic level, with initial momentum such that it travels past an avoided crossing. What is the shape and size of the second component of $\psi_{0}(x, t)$, to leading order, long after passing the avoided crossing?

Time-dependent first order perturbation theory gives a straightforward answer. We write $H^{ \pm}=-\frac{\varepsilon^{2}}{2} \partial_{x}^{2} \pm \rho(x)+$ $d(x)$ for the Hamiltonian generating the uncoupled dynamics in the upper (respectively, lower) band. To lowest order in $\varepsilon$, the first component $\psi_{0}^{+}$of $\psi_{0}$ evolves by the BOA, $\psi_{0}^{+}(t)=e^{-(i / \varepsilon) t H^{+}} \phi$, where we have chosen the initial condition $\phi$ at time $t=0$. Thus, to leading order,

$$
\psi_{0}^{-}(t)=-i \varepsilon \int_{-\infty}^{t} e^{-(i / \varepsilon)(t-s) H^{-}} K_{1} \psi_{0}^{+}(s) d s
$$

with $K_{1}=\kappa_{1}(x)\left(\varepsilon \partial_{x}\right)$. However, (3) gives very little insight into the nature of the transmitted wave function. In particular, it appears to be of order $\varepsilon$, while the true $\psi_{0}^{-}$is 
expected to be exponentially small in $\varepsilon$ away from the transition region [13].

A better picture is obtained by studying superadiabatic representations [14]. Like the adiabatic representation, these are implemented by unitaries $U_{n}$ acting in $L^{2}\left(\mathbb{R}, \mathbb{C}^{2}\right)$, which are now pseudodifferential operators rather than multiplications. In [15], a general recursive scheme for computing $U_{n}$ is given. When $U_{n}$ is the $n$th superadiabatic unitary, and $H_{n}=U_{n}^{-1} H U_{n}$, then to leading order

$$
H_{n}=-\frac{\varepsilon^{2}}{2} \partial_{x}^{2} \boldsymbol{I}+\left(\begin{array}{cc}
\rho(x)+d(x) & \varepsilon^{n+1} K_{n+1}^{+} \\
\varepsilon^{n+1} K_{n+1}^{-} & -\rho(x)+d(x)
\end{array}\right) .
$$

Therefore, with $\psi_{n}=U_{n} \psi$, (3) can be replaced by

$$
\psi_{n}^{-}(t)=-i \varepsilon^{n} \int_{-\infty}^{t} e^{-(i / \varepsilon)(t-s) H^{-}} K_{n+1}^{-} e^{-(i / \varepsilon) s H^{+}} \phi d s .
$$

Equation (5) correctly describes the wave function in the $n$th superadiabatic representation. For suitable ("optimal") $n$, (5) corresponds to a single crossing transition, where $\psi_{n}^{-}(t)$ builds up monotonically. This was shown in a special case [16], and we expect it to hold in general. However, the adiabatic or even diabatic description of the transmitted wave function is not, in general, easily derived from (5): $U_{n}$ is a pseudodifferential operator and is neither easy to analyze nor to implement on a computer. This is not surprising, as, e.g., near the avoided crossing $\psi_{0}^{-}$displays wild oscillations of order $\varepsilon$, and we have no reason to expect any simple description.

The situation is different in the scattering regime. When $V$ is approximately constant, $U_{n}$ and $U_{0}$ agree up to small errors. Therefore (5) can be used to compute the transmitted wave function after an avoided crossing, sufficiently far away from the crossing, in the adiabatic representation. But even then, its usefulness depends on our ability to understand the $n$th superadiabatic coupling operator $K_{n+1}^{-}$ to leading order. In the present context, this can be done [16]: $K_{n}^{-}$is the Weyl quantization

$$
K_{n}^{-} \psi(x)=\frac{1}{2 \pi \varepsilon} \int_{\mathbb{R}^{2}} d \xi d y \kappa_{n}^{-}\left(\frac{x+y}{2}, \xi\right) e^{(i / \varepsilon) \xi(x-y)} \psi(y)
$$

of the symbol $\kappa_{n}^{-}$. The latter is determined by a recursive system of differential equations, which follows from the established scheme [15] by a choice of basis. It has been derived in [16] for the case $d(x)=0$; the extension to $d(x) \neq 0$ is straightforward. To leading order, $K_{n}$ is a differential operator of order $n$, with symbol $\kappa_{n}(p, q)=$ $\sum_{j=0}^{n} p^{j} \kappa_{n, n-j}(q) . \kappa_{n, j}(q)$ can be calculated explicitly [16] for all $n$ and $j$, but at present we only control the asymptotics of $\kappa_{n, 0}(q)$. For moderate $n$, the latter dominate, but in the limit $n \rightarrow \infty$, they do not. Nevertheless, we approximate (6) by keeping only $\kappa_{n, 0}(q)$. This appears dubious at first, as typically $n \sim \varepsilon^{-1}$, but it can be justified when the incoming momentum is large [16]. In practice, even for moderate incoming momenta our theory gives excellent results; for those cases where it does become inaccurate, the transition probability is extremely small. We will comment more on this later on.

The asymptotics of $\kappa_{n, 0}^{-}$can be determined in the following generic case. Assume an avoided crossing at $x=0$. Write $\rho^{2}(q)=\delta^{2}+g(q)^{2}$ with $g(0)=0$ and $g$ analytic. Since $g^{2}$ is quadratic at 0 , a Stokes line [i.e., a curve where $\operatorname{Im}(\rho)=0]$ crosses the real axis perpendicularly. For $\delta$ not too large, following this line into the complex plane leads to a pair of complex conjugate points $q_{\delta}, q_{\delta}^{*}$ where $\rho$ has complex zeroes. Berry and Lim [5] show that, in the natural scale $\tau(q)=2 \int_{0}^{q} \rho(r) d r$, these zeroes give rise to a pair of complex first order singularities of the adiabatic coupling function $\kappa_{1}$. Near $q=0, \quad \kappa_{1}(q)=(i \rho(q) / 3)([\tau(q)-$ $\left.\left.\tau_{\delta}^{*}\right]^{-1}-\left[\tau(q)-\tau_{\delta}\right]^{-1}+\kappa_{r}[\tau(q)]\right)$, with $\tau_{\delta}=\tau\left(q_{\delta}\right) . \kappa_{r}$ has no singularities of order $\geq 1$ for $|\tau| \leq\left|\tau_{\delta}\right|$. Solving the recursive equations for $\kappa_{n}^{-}$involves taking high derivatives of $\kappa_{1}$; by the Darboux principle [6,17], the complex singularities $\tau_{\delta}$ and $\tau_{\delta}^{*}$ closest to the real axis dominate the asymptotics. It follows $[6,16]$ that to leading order, with $h_{n}(\tau)=i\left(\tau-\tau_{\delta}^{*}\right)^{-n}-i\left(\tau-\tau_{\delta}\right)^{-n}$,

$$
\kappa_{n, 0}^{-}(q)=\frac{i^{n}}{\pi} \rho(q)(n-1) ! h_{n}[\tau(q)] .
$$

A direct calculation now gives the Weyl quantization of $p^{n} \kappa_{n, 0}^{-}(q) \quad$ as $\quad K_{n, 0}^{-}=\sum_{j=0}^{n}\left(\begin{array}{l}n \\ j\end{array}\right)\left(\frac{\varepsilon}{2 i}\right)^{j}\left[\partial^{j} \kappa_{n, 0}^{-}(x)\right]\left(-i \varepsilon \partial_{x}\right)^{n-j}$. Relevant values of $n$ are of order $1 / \varepsilon$. For these, $\kappa_{n}^{-}$and its derivatives are concentrated in a $\sqrt{\varepsilon}$ neighborhood of $x=0$. Thus the action of $K_{n, 0}^{-}$on any function without support near zero is negligible. As semiclassical wave packets are of width $\sqrt{\varepsilon}$ and travel with speed of order one under the dynamics, the dominant contribution to (5) comes from times $s$ where the upper band wave packet is very close to the transition point $x=0$. When we choose the coordinates so that the initial condition $\phi$ is concentrated at $x=0$, relevant times $s$ are of order $\sqrt{\varepsilon}$.

Using this, we now show that under the integral in (5), the adiabatic potentials $a^{ \pm}(x)= \pm \rho(x)+d(x)$ entering $H^{ \pm}$can be replaced by their first order approximations at 0 , given by $a_{1}^{ \pm}(x)= \pm \delta+\lambda x$ : we write $g^{ \pm}=a^{ \pm}-a_{1}^{ \pm}$ and $H_{1}^{ \pm}=-\varepsilon^{2} \partial_{x}^{2} / 2+a_{1}^{ \pm}$, and obtain $e^{-(i / \varepsilon) s H^{ \pm}}-$ $e^{-(i / \varepsilon) s H_{1}^{ \pm}}=-\frac{i}{\varepsilon} \int_{0}^{s} e^{-(i / \varepsilon)(s-r) H_{1}^{ \pm}} g^{ \pm} e^{-(i / \varepsilon) r H^{ \pm}} d r$. As $g^{ \pm}$ is quadratic near $x=0, g^{ \pm} e^{-(i / \varepsilon) r H^{ \pm}} \phi$ is of order $\varepsilon$ in a $\sqrt{\varepsilon}$ neighborhood of 0 . There, the right-hand side above is bounded by the length of the integration region $\sqrt{\varepsilon}$, while outside that neighborhood, application of $K_{n}^{-}$yields a negligible result. As the image under $K_{n}^{-}$is again sharply peaked around 0 , a similar argument for $e^{(i / \varepsilon) s H^{-}}$shows the claim.

We now switch to the Fourier representation. The correct scaling is retained by the semiclassical Fourier transform

$$
\hat{f}^{\varepsilon}(k)=\frac{1}{\sqrt{2 \pi \varepsilon}} \int e^{-(i / \varepsilon) k q} f(q) d q .
$$

We define $\hat{K}_{n}$ through $\hat{K}_{n} \hat{\psi}^{\varepsilon}={\widehat{K_{n}}}^{\varepsilon}$, and a direct calcu- 
lation using (6) gives

$$
\hat{K}_{n, 0}^{-} f(k)=\frac{1}{\sqrt{2 \pi \varepsilon}} \int_{\mathbb{R}} d \eta{\widehat{\kappa_{n, 0}^{-}}}^{\varepsilon}(k-\eta)\left(\frac{\eta+k}{2}\right)^{n} f(\eta) .
$$

We use (8) on (7), and change variables from $q$ to $\tau$ in the integral. Using $d \tau=2 \rho(q) d q$ we get

$$
\widehat{\kappa_{n, 0}^{-}} \varepsilon(k)=\sqrt{\frac{2}{\pi^{3} \varepsilon}}(n-1) ! \int e^{(i / \varepsilon) k q(\tau)} h_{n}(\tau) d \tau
$$

Since $\rho$ has a minimum $\delta$ at $q=0$, we have $q(\tau)=$ $\tau /(2 \delta)+\mathcal{O}\left(\tau^{3}\right)$. The third and higher order terms are negligible since $h$ is concentrated around $q=0$, and an application of the residual theorem gives

$$
\widehat{\kappa_{n, 0}^{-}} \varepsilon(k)=i \frac{\sqrt{2} \delta}{\sqrt{\pi \varepsilon}(2 \delta)^{n}}\left(\frac{k}{\varepsilon}\right)^{n-1} e^{(|k| / 2 \delta \varepsilon) i \tau_{\delta}} .
$$

For the propagators, the Avron-Herbst formula [18] gives

$$
e^{-(i / \varepsilon) s \hat{H}_{1}^{ \pm}}=e^{-i\left(\lambda^{2} s^{3} / 6 \varepsilon\right)} e^{\lambda s \partial_{k}} e^{-(i / 2 \varepsilon)\left[\left(k^{2} \pm 2 \delta\right) s+\lambda k s^{2}\right]}
$$

The shift terms of $e^{ \pm(i / \varepsilon) s \hat{H}_{1}^{ \pm}}$, acting on $\eta$, respectively $k$, replace the term $(\eta+k)^{n}$ with $(\eta+k+2 \lambda s)^{n}$ in (9), and leave functions of $\eta-k$ unchanged. We abbreviate $a=$ $\eta+k, b=\eta-k$, and find

$$
\begin{aligned}
\widehat{\psi_{n}^{-}} \varepsilon(k, t)= & \frac{1}{4 \pi \varepsilon} e^{-(i / \varepsilon) t H^{-}} \int_{-\infty}^{t} d s \int d \eta(a-2 \lambda s)^{n+1} \\
& \times\left(\frac{b}{4 \delta}\right)^{n} e^{i\left(\tau_{\delta} / 2 \delta \varepsilon\right)|b|} e^{(i / 2 \varepsilon)\left[(a b-4 \delta) s+\lambda b s^{2}\right]} \hat{\phi}^{\varepsilon}(\eta) .
\end{aligned}
$$

This equation describes, to leading order, the transmitted wave function at all times $t$ in the $n$th superadiabatic representation, for $n$ large enough. When $t$ is significantly larger than $\sqrt{\varepsilon}$, the domain of integration in $s$ can be extended to $\mathbb{R}$. We write $(a-2 \lambda s)^{n+1}=a^{n+1} \exp [(n+$ 1) $\log (1-2 \lambda s / a)]$, and expand the logarithm to second order in $s$. Gaussian integration in $s$ yields

$$
\widehat{\psi_{n}^{-}} \varepsilon(k, t)=\frac{e^{-(i / \varepsilon) t H^{-}}}{4 \pi \varepsilon} \int d \eta \frac{a \sqrt{2 \pi \varepsilon a^{2}}}{\sqrt{4 \mu \lambda^{2}+i \lambda a^{2} b}}\left(\frac{a b}{4 \delta}\right)^{n} e^{i\left(\tau_{\delta} / 2 \delta \varepsilon\right)|b|} \exp \left(\frac{[4 \mu \lambda-i a(a b-4 \delta)]^{2}\left(4 \mu \lambda-i a^{2} b\right)}{8 \varepsilon \lambda\left[(4 \mu \lambda)^{2}+\left(a^{2} b\right)^{2}\right]}\right) \hat{\phi}^{\varepsilon}(\eta),
$$

with $\mu=\varepsilon n$. Equation (10) reduces our task to the calculation of a one-dimensional integral, but some tricky points remain: the choice of $n$ is not obvious, and one oscillatory integral has to be calculated for each desired value of $k$. A systematic analysis of (10) is currently in progress; here we restrict to an important special case. If, e.g., for symmetry reasons, the slope $\lambda$ of the potential surfaces is much smaller than the energy gap at the crossing point, Laplace's method can be applied to the integral in (10), with respect to the small parameter $\lambda$. Both stationary phase and maximal absolute value are attained when $a b=$ $4 \delta$. There, the dependence of (10) on $n$ disappears, and we obtain the main result of the present Letter: for times $t \gg$ $\sqrt{\varepsilon}$, the transmitted wave packet is given by

$$
\widehat{\psi^{-}} \varepsilon(k, t)=e^{-(i / \varepsilon) t H^{-}} 1_{\left\{k^{2}>4 \delta\right\}} \frac{v+k}{2|v|} e^{i\left(\tau_{\delta} / 2 \delta \varepsilon\right)|k-v|} \hat{\phi}^{\varepsilon}(v),
$$

where $v=v(k, \delta)=\operatorname{sgn}(k) \sqrt{k^{2}-4 \delta}$. We write $\tau_{\delta}=$ $\tau_{r}+i \tau_{c} ; \tau_{c}$ determines the LZ-transition amplitude, while $\tau_{r}$ induces a phase shift.

The physical significance of (11) becomes clear when we take $\phi$ as a plane wave with momentum $p_{0}$. Then $\hat{\phi}^{\varepsilon}=$ $\delta_{p_{0}}$, and $\psi^{-}$is again a plane wave. Its momentum $k\left(p_{0}\right)=$ $\operatorname{sgn}\left(p_{0}\right)\left(p_{0}^{2}+4 \delta\right)^{1 / 2}$ is determined by energy conservation. Its amplitude contains a LZ-type exponentially small term with rate $-(2 \delta \varepsilon)^{-1} \tau_{c}\left|k\left(p_{0}\right)-p_{0}\right|$. The rate increases towards zero when $p_{0}$ grows: fast parts of the wave function are less adiabatic, and therefore more likely to make the transition. For large $p_{0}$ we have $k\left(p_{0}\right)-p_{0} \approx 2 \delta / p_{0}$, and we recover the classical LZ-transition rate $-\left(p_{0} \varepsilon\right)^{-1} \tau_{c}$. Incidentally, the regime of large $p_{0}$ is also where (11) becomes asymptotically exact, but we emphasize that for moderate $p_{0}$, numerical tests show the transition rates obtained from (11) to be far superior to the classical LZ ones. To summarize, nonadiabatic transitions decouple in momentum space. Each incoming momentum makes a separate, single step LZ-type transition, with the outgoing momentum determined by energy conservation. The transition rate, phase shift and prefactor approach the classical LZ ones for large momenta, but differ for moderate momenta, where they are well approximated by those given in (11).

Our results suggest a simple algorithm for computing nonadiabatic transitions: evolve the upper band wave function according to the decoupled evolution until an avoided crossing is detected, e.g., by maximality of the adiabatic coupling functions. There use (11) [or, if necessary, (10)] with $t=0$ to produce a transmitted wave packet $\widehat{\psi_{n}^{-}}(k)$ at the transition point. Propagate this according to the decoupled lower band dynamics. This is numerically much cheaper than the fully coupled dynamics, where the time step has to be extremely short due to the smallness of the required final result.

For a numerical demonstration of (11), we choose $X(x)=\delta, \quad Z(x)=\alpha \tanh x+\beta x^{2} / \cosh x$, and $d(x)=$ $\lambda \tanh x$, with $\alpha=1 / 2, \beta=1 / 4$, and $\delta=1 / 2 . \tau_{\delta}$, known here analytically, can in general be easily obtained numerically as a line integral along $s q_{\delta}, 0 \leq s \leq 1$. We find $\tau_{1 / 2} \approx-0.13099+0.58917 i$. We are interested in situations where $\lambda \ll \alpha$ and choose $\lambda=0.1$ [cf. Fig. 1(a)].

For solving (1), the initial condition and time axis are again chosen such that the wave packet $\psi_{0}^{+}(t)$ reaches the avoided crossing (located at $x=0$ ) at time $t=0$. We treat 
a)

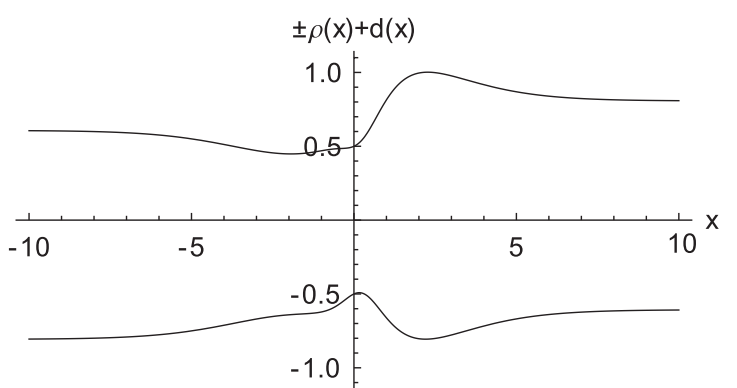

b)
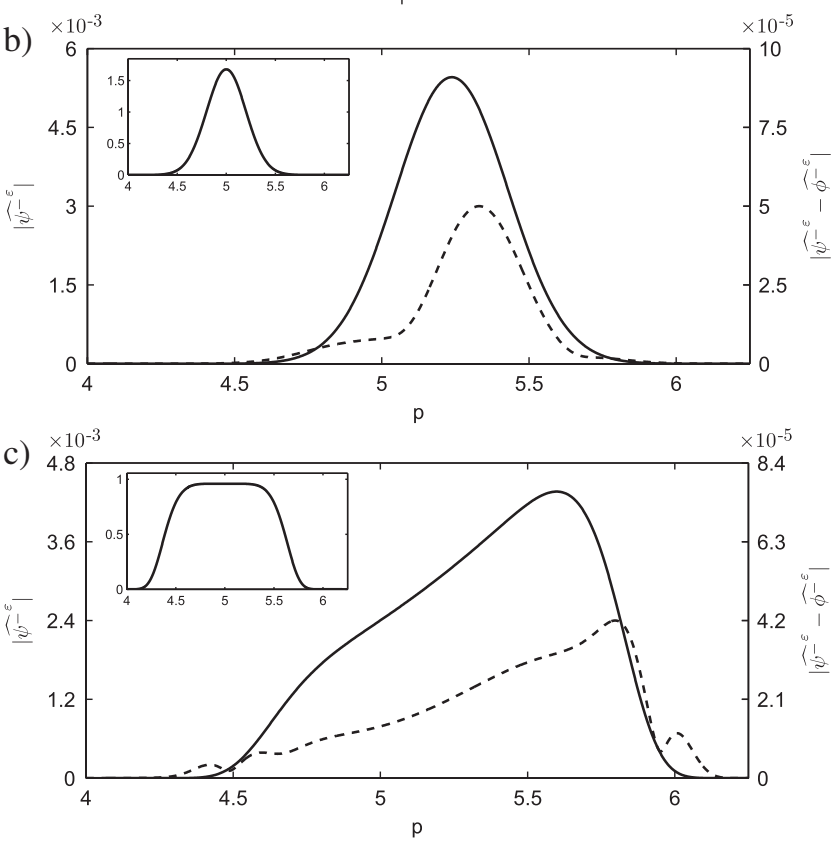

FIG. 1. Numerical illustration of formula (11): (a) shows the potential energy surfaces used. (b) and (c) show the numerical results for Gaussian, respectively, non-Gaussian incoming wave function (embedded graph). Displayed is the absolute value of the transmitted wave functions ${\widehat{\psi^{-}}}^{\varepsilon}$ as calculated using (11) (solid lines, left axis), along with the absolute value of the error between $\widehat{\psi^{-\varepsilon}}$ and the exact numerical calculation $\widehat{\phi^{-\varepsilon}}$ (dashed lines, right axis).

the standard case of a Gaussian, $\widehat{\phi}_{g}^{\varepsilon}(p)=\frac{1}{N_{1}} \exp [-(p-$ $\left.\left.p_{0}\right)^{2} /\left(2 \sigma^{2} \varepsilon\right)\right]$, and the case ${\widehat{\phi_{\mathrm{ng}}}}^{\varepsilon}(p)=\frac{1}{N_{2}} \exp [-(p-$ $\left.\left.p_{0}\right)^{6} /\left(2 \sigma^{2} \varepsilon\right)\right] . N_{1}$ and $N_{2}$ are $L^{2}$ normalizations, and we take $p_{0}=5, \varepsilon=1 / 50$, and $\sigma^{2}=2$.

The numerically exact solutions ${\widehat{\phi^{-}}}^{\varepsilon}$ are obtained using a standard symmetric Strang splitting in MATLAB. We evolve $\phi$ backwards in time with the decoupled dynamics, until it is concentrated in a region where the energy levels are essentially flat. The result is taken as the initial condition for the fully coupled dynamics, run until a time $t_{*}>0$ where again the energy levels are flat; i.e., the lower component $\left\|\psi_{0}^{-}\left(t_{*}\right)\right\|$ is constant in time. $\psi_{0}^{-}\left(t_{*}\right)$ is then evolved backwards to $t=0$ with the uncoupled dynamics, where its Fourier transform is compared with (11) for $t=$
0 . Numerical convergence is assured by repeated reduction of the time step. The results are shown in Fig. 1.

For both incoming wave functions, the relative error is less than $1 \%$, over the full interval where the transmitted wave function is essentially supported. The transition probability $\left\|\psi^{-}\right\|^{2}$ in the Gaussian case computes to $9.97 \times 10^{-6}$ using (11), with a relative error of around $1.4 \%$. For comparison, the best results obtained by surface hopping algorithms have an error of around 3\% [7].

In addition to the examples presented here, we tested a wide range of parameters. Provided that we keep $\lambda$ small enough, all results are good to within a few percent. They deteriorate either when $\varepsilon$ (and thus $\left\|\psi^{-}\right\|$) becomes too large and we leave the adiabatic regime, or when $p_{0}$ (and thus $\left.\left\|\psi^{-}\right\|\right)$gets too small and our approximation of keeping only $\kappa_{n, 0}$ in (6) becomes inaccurate. In the first case, we found good agreement up to transition probabilities $\left\|\psi^{-}\right\|^{2} \approx 10^{-2}$, and in the second case down to $\left\|\psi^{-}\right\|^{2} \approx$ $10^{-20}$. The latter suggests that unless nonadiabatic transitions are so small that they are likely to be physically irrelevant, they are accurately described by (11).

We thank Stefan Teufel for useful comments. V. B. is supported by the EPSRC grant EP/D07181X/1.

[1] A. H. Zewail, Femtochemistry: Ultrafast Dynamics of the Chemical Bond (World Scientific, New York, 1994).

[2] R. Schoenlein et al., Science 266, 422 (1994).

[3] J. V. Neumann and E. Wigner, Phys. Z. 30, 467 (1929).

[4] D. Zener, Proc. R. Soc. A 137, 696 (1932).

[5] M. Berry and R. Lim, J. Phys. A 26, 4737 (1993).

[6] V. Betz and S. Teufel, Commun. Math. Phys. 260, 481 (2005).

[7] C. Lasser and T. Swart, J. Chem. Phys. 129, 034302 (2008).

[8] C. Lasser, T. Swart, and S. Teufel, Commun. Math. Sci. 5, 789 (207).

[9] A. Voronin, J. Marques, and A. Varandas, J. Phys. Chem. A 102, 6057 (1998).

[10] J. Tully, J. Chem. Phys. 93, 1061 (1990).

[11] H. Nakamura, Nonadiabatic Transition (World Scientific, Singapore, 2002), ISBN 978-981-02-4719-5.

[12] G. A. Hagedorn and A. Joye, Ann. Henri Poincaré 6, 937 (2005).

[13] G. Hagedorn and A. Joye, Commun. Math. Phys. 223, 583 (2001).

[14] M. Berry, Proc. R. Soc. A 429, 61 (1990).

[15] S. Teufel, in Adiabatic Perturbation Theory in Quantum Dynamics, Lecture Notes in Mathematics Vol. 1821 (Springer-Verlag, Berlin, 2003), ISBN 3-540-40723-5.

[16] V. Betz, B. D. Goddard, and S. Teufel, Proc. R. Soc. A 465, 3553 (2009).

[17] R. Dingle, Asymptotic Expansions: Their Derivation and Interpretation (Academic Press, New York, 1973).

[18] J. Avron and I. Herbst, Commun. Math. Phys. 52, 239 (1977). 